\title{
Quelques résultats théoriques récents concernant les écoulements des nappes d'eau souterraines ${ }^{*}$
}

(Suite et fin)

\author{
A few recent theoretical results \\ concerning ground water flow (*)
}

\author{
PAR R. MEYER
}

INGENIEUR AU LABOHATOIAE DAUPHINOIS D'HYDRAULIQUE, GRENOBLE

La présente partie de cet article contient essenticllement les justificalions théoriques des assertions énoncées précédemment et relatives aux nappes de grande épaisseur. De plus, on montre clairement que les termes « grande 》 et « petite » épaisseur sont en réalité impropres et qu'il conviendrait plutôt de dire: "distances horizontales petites $\gg$ ou $\ll$ grandes devant l'épaissear ». La théorie des nappes de grande chaisseur s'applique donc toujours vers les bords des nappes à leur surface libre; par contre, l'approximation de Dupuit peut ne jamais s'appliquer si les dimensions horizontales globales sont petites devant l'épaisseur (cas des digues par exemple).

On montre aussi que les caractéristiques des nappes (perméabilité, porosité, obstacles, etc.) n'influencent que peu les forces exercées par l'eau sur les ouvrages situés dans les nappes ou sur leur bord. Ceci est particulièrement important; en effet, dans les nappes habituelles, ces caractéristiques sont essentiellement variables d'un point à un antre point (même très voisin du premier). De plus, ces caractéristiques sont souvent difficiles dà mesurer voire même à définir.

Fin résumé, il y a deux cas d'études possibles: ítudes globales d'une riappe par une méthode du type «approximation de Dupuit » et en se servant des caractéristiques moyennes mesurcies par des méthodes mettant en jeu des parties importantes des nappes et études locales par une méthode du type « n'appes à grande épaisseur $\gg \dot{a}$ la condition que les caractéristiques lointaines n'interviennent pas et que les caractéristiques locales proches interviennent peu.
This section of the article is fundamentally concerned with theoretical proofs of statements made previously in connection with deep ground water. Furthermore it is clearly shown that the expressions "deep" and "shallow" are unsatisfactory and that it is better to suy "small horizontal distances" or "large compared with depth". The theory of deep groun'd water can always be applied at the free surface near a boundary. On the other hand cases occur where Dupait's approximation' cannot be applied, for example when the maximum horizontal dimensions are small compared with the depth (e.g. dykes).

It is also shown that conditions such as permeability, porosity, obstacles, etc. have Iittle effect upon the forces which the water exerts upon structures surrounded by, or boudaries of ground water. This is very important for, in most cases, such conditions vary fundamentally from point to point even when these points are close together, Furthermore it is often difficult to measure these conditions, let alone define them.

In shorl, there are two possible means of innestigation:

An overall investigation of a ground wale. region by a "Dupuit Approximation" method and using mean measurements of condilions, obtained by means which reflect the effect of a large expanse of ground water; a localized investigation' using a "thick layer of ground water" method where distant conditions and conditions in the immediate vicinity have little effect.

$\left(^{*}\right)$ Cf. La Houille Blanche, $\mathrm{n}^{\circ} 1,1955 ; \mathrm{n}^{\circ} 5,1955$. 


\section{CHAPITRE III. - CONSIDÉRA'TIONS THÉORIQUES} (Suite)

3-6. - NAPPES DE GRANDE ÉPAISSEUR.

3-7. - SURPRESSTONS SUR LES MURS DE QUAI DUES A LA MARÉE.

3-8. - PHÉNOMẼNES NON SINUSOIDAUX DANS LES NAPPES DE GRANDE EPPAISSEUR.

3-9. - CONCLUSIONS DU CHAPITRE III.

\section{CHAP I T R E I I I}

\section{CONSIDERATIONS THEORIQUES}

(Suite)

\section{3-6. - Nappes de grande épaisseur.}

Reprenons les équations du paragraphe 3-1 : $\Delta_{3} h=\mathbf{0}$ dans le milieu poreux; $\frac{\partial h}{\partial z}+\frac{m}{\mathrm{~K}} \frac{\partial h}{\partial t}=0$ à la surface libre; $\frac{\partial h}{\partial n}=0$ sur les obstacles.

Ces équations ne peuvent être intégrées que dans un nombre de cas très restreint. Il faut pour cela :

1) Que le milieu soit homogène et limité vers le bas par une couche impermćable horizontale;

2) Que les autres parois soient verticales et entièrement constituées sur chaque verticale soit d'obstacles, soit de surface de contact entre l'eau souterraine et de l'eau libre:

3) Que leur tracé en plan soit formé :

a) ou bien d'ellipses et hyperboles homofocales;

b) ou bien de cercles concentriques et de droites passant par le centre;

c) ou bien de droites orthogonales;

d) ou bien des paraboles homofocales;

4) Que les angles vus de l'intérieur de l'éconlement et formés par les divers arcs soient de $90^{\circ}$ (et non de $270^{\circ}$ );

5) Que chaque arc soit un obstacle ou une surface de contact avec l'eau libre.
Dans tous les autres cas, il faut faire des approximations. Aux paragraphes 3-3 et 3-4, nous avons décrit certains calculs faits avec l'approximation de Dupurt applicable à une certaine distance des limites et quand l'épaisseur de la nappe est faible. Nous allons maintenant faire une autre approximation, celle (en quelque sorte inverse) de la nappe de grande épaisseur, qui revient à supposer l'épaisseur de la nappe infiniment grande (fig. 51).
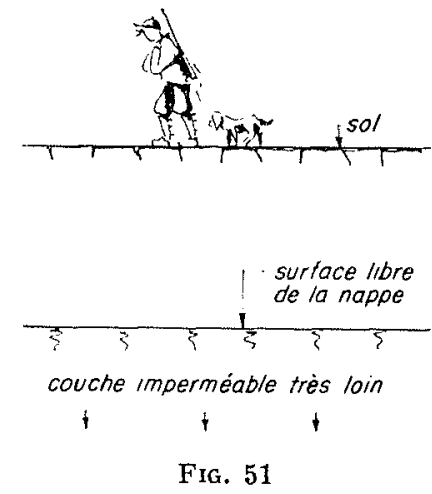

Etudions d'abord les écoulements sinusoïdaux en posant, comme au paragraphe 32 :

$h=\mathcal{R}\left[\mathrm{H} e^{j \omega t}\right]$, d'où la condition à la surface libre:

$$
\frac{\partial H}{\partial z}+\frac{m}{K} j \omega H=0
$$


Si on pose dans tout le milieu :

$$
\frac{\partial \mathbf{H}}{\partial z}+\frac{m}{\mathrm{~K}} j \omega \mathbf{H}=\Phi
$$

La fonction $\Phi$ satisfait à l'équation :

$$
\Delta_{3} \Phi=0
$$

dans le milieu et aux conditions :

$$
\Phi=0 \text { à la surface libre }
$$

et :

$$
\Phi=\frac{\partial \mathbf{H}}{\partial z}+\frac{m}{\mathbf{K}} j \omega \mathbf{H}=\text { "donnée " }
$$

sur les surfaces de contact avec l'eau libre.

Pour que la condition à la limite sur un obstacle imperméable soit exprimable en fonction de $\Phi$, il faut que cet obstacle soit un cylindre à génératrices verticales; alors :

$\frac{\partial \Phi}{\partial n}=\frac{\partial}{\partial n}\left(\frac{\partial H}{\partial z}\right)+\frac{m}{K} j \omega \frac{\partial H}{\partial n}=\frac{\partial}{\partial z}\left(\frac{\partial H}{\partial n}\right)=0$

La dernière égalité est vraie parce que l'accroissement $\langle d z »$ est le long de la génératrice de la paroi. Nous avons alors ramené le problème avec des conditions aux limites mixtes à un problème n'ayant que des conditions aux limites du type :

$$
\frac{\partial \Phi}{\partial n}=0
$$

ou :

$$
\Phi=\Phi_{0}=\text { "donnée " }
$$

On peut donc résoudre un grand nombre de problèmes plans et certains problèmes à trois dimensions. Connaissant $\Phi$, on en déduit $\mathbf{H}$ par :

$$
\frac{\partial \mathrm{H}}{\partial z}+\frac{m}{\mathrm{~K}} \boldsymbol{j} \omega \mathrm{H}=\Phi
$$

Cette méthode est analogue à celle déjà employée au paragraphe 34 pour les images d'une source par rapport à une paroi. On peut généraliser cette méthode au cas où les parois du milieu noreux ne sont pas verticales, mais inclinées. Il s'agit essentiellement de trouver une fonction Tliée linéairement à $\mathrm{H}$ et aux dérivées nartielles de $\mathrm{H}$ et telle que $\Phi=0$ a la surface libre et $(\partial \Phi / \partial n)=0$ sur les parois imperméables. Une méthode très intéressante a été publiée nar Fritz John [87. La clé du problème se trouve essentiellement page 158 . équation $(1 ; 32)$. Nous n'entrons pas dans la discussion de ces cas qui sont nettement plus délicats que ceux qui seront exposés ici, tout en n'apportant que des résultats semblables, sans notions vraiment nouvelles.
Donnons un exemple de la méthode. Soit à déterminer les ondes produites dans un milieu poreux par une variation sinusoïdale d'un plan

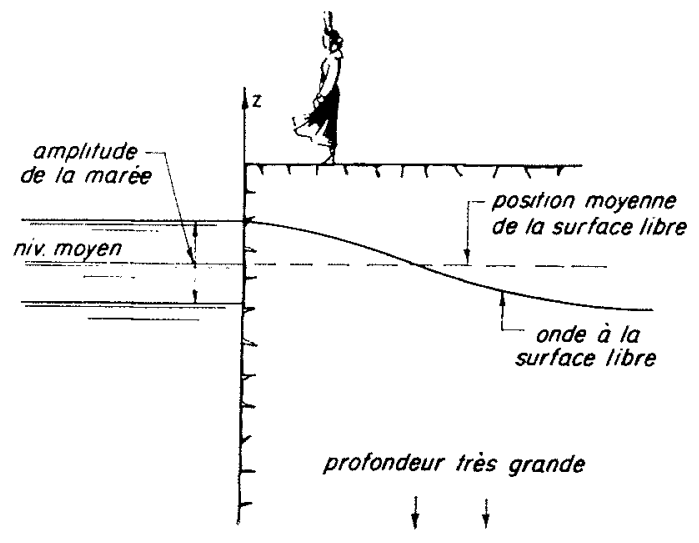

FiG. 52

d'eau libre, comme indiqué sur la figure 52 (marée).

Dans ce cas, on a :

$$
h=\mathrm{H}_{0} e^{j \omega t} \operatorname{sur} x=0 ; z<0
$$

$\Phi$ est déterminé par :

$$
\begin{aligned}
\Delta_{2} \Phi & =0 \\
\Phi & =0 \text { sur } z=0 ; x>0 \\
\Phi & =\frac{m}{\mathrm{~K}} j \omega \mathrm{H}_{0} \text { sur } x=0 ; z<0 .
\end{aligned}
$$

En plus, $\Phi \rightarrow 0$ pour $x \rightarrow \infty$.

La solution $\Phi$ est évidente et se déduit du réseau isotherme correspondant $\dot{a} \mathrm{Z}=j \log \zeta$, avec $Z=\Phi+j \Psi$ et $\zeta=x+j z$ (fig. 53).

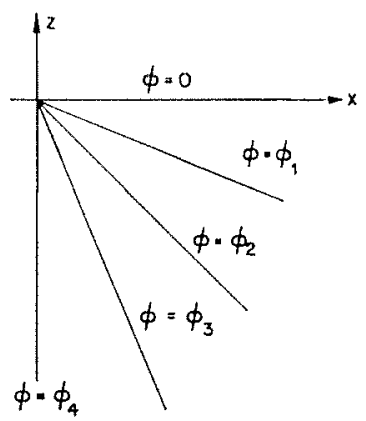

FiG. 53

Donc :

$$
\Phi=\frac{2}{\pi} \frac{m}{\mathrm{~K}} j \omega \mathrm{H}_{0} \operatorname{Arctg} \frac{z}{x}
$$


D'où :

$\frac{\partial \mathrm{H}}{\partial z}+\frac{m}{\mathrm{~K}} j \omega \mathrm{H}=\frac{2}{\pi} \frac{m}{\mathrm{~K}} j \omega \mathrm{H}_{0} \operatorname{Arctg} \frac{z}{x}$

On en déduit :

$$
\begin{gathered}
\mathrm{H}=\frac{\pi}{2} j \omega \frac{m}{\mathrm{~K}} \mathrm{H}_{0} e^{-j \omega(m / \mathrm{K}) z} \int_{0}^{z} e^{j \omega(m / \mathrm{K}) u} \operatorname{Arctg} \frac{u}{x} d u \\
+\frac{2}{\pi} \mathrm{j} \omega \frac{m}{\mathrm{~K}} \mathrm{H}_{0} e^{-j \omega(m / \mathrm{K}) z} \cdot f(x)
\end{gathered}
$$

Posons :

$$
\frac{m}{\mathrm{~K}} \omega z=\zeta ; \frac{m}{\mathrm{~K}} \omega x=\xi ; \frac{m}{\mathrm{~K}} \omega u=v
$$

L'expression trouvée vérifie bien :

$$
\frac{\partial \mathrm{H}}{\partial z}+j \omega \frac{m}{\mathrm{~K}} \mathrm{H}=\mathbf{0}
$$

à la surface libre, comme on s'en assure facilement, mais pour satisfaire à $\Delta \mathbf{H}=0$, il faut que $f(\xi)$ satisfasse à $f^{\prime \prime}-f=(1 / \xi)$.

D'où :

$$
f=-\frac{1}{2} e^{\xi} \int_{\xi_{1}}^{\xi} \frac{e^{-w}}{w} d w-\frac{1}{2} e^{-\xi} \int_{\xi_{2}}^{\xi} \frac{e^{-w}}{w} d w
$$

En plus, il faut que $H$ satisfasse aux conditions $\mathrm{H}=0$ en $x=\infty$ et $\mathrm{H}=\mathrm{H}_{0}$ en $x=0$. Ceci revient à exiger que $f(\infty)=0$ et $f(0)=1$. La première condition donne $\xi_{1}=\infty$, la deuxième :

$$
-2=\int_{-\xi_{n}}^{\infty}-\frac{e^{-w}}{w} d w
$$

D'où :

$$
\begin{gathered}
\mathrm{H}=\frac{2}{\pi} j \mathrm{H}_{0} e^{-j \zeta} \int_{0}^{\zeta} e^{j e} \operatorname{Arctg} \frac{v}{\xi} d v+\frac{1}{\pi} j \mathbf{H}_{0} e^{-j \xi} \\
{\left[e^{-\xi} \int_{-\xi}^{\infty} e^{-w} d w-e^{\xi} \int_{\xi}^{\infty} e^{-w} w d w+\mathrm{H}_{0} e^{-j \zeta} e^{-\xi}\right.}
\end{gathered}
$$

La surface libre est donnée par $\zeta=0$ :

$$
\begin{aligned}
\mathrm{H}=\frac{1}{\pi} j \mathrm{H}_{0}\left[e^{-\xi}\right. & \int_{-\xi}^{\infty} \frac{e^{-w}}{w} d w \\
& \left.-e^{\xi} \int_{\xi}^{\infty} \frac{e^{-w}}{w} d w\right]+\mathrm{H}_{0} e^{-\xi}
\end{aligned}
$$

Rappelons que les intégrales intervenant sont des fonctions dont il existe des tables (voir par exemple [5]).

La surface libre ne contient pas d'ondes comme dans le cas des nappes d'épaisseur finie. En fait nous avons vi que si la distance $x$ est plus grande que l'épaisseur de la nappe, l'approximation de Dupurt rend bien compte du phénomène. L'approximation nappe de grande épaisseur ne peut donc être valable que si la distance $x$ est nettement inférieure à l'épaisseur de la nappe. Nous trouvons ici cet écoulement transitoire dans l'espace dont il était question avant.

L'approximation est donc surtout intéressante pour étudier ce qui se passe près des limites, obstacles ou d'autres irrégularités. A titre d'exemple, nous étudierons les surpressions derrière les murs de quai.

D'une façon générale, le problème se scinde en deux parties :

a) Recherche de la fonction $\Phi$;

b) Recherche de $\mathrm{H}$ par la formule :

$\mathrm{H}(j \omega)=j \omega \frac{m}{\mathrm{~K}} e^{-j \omega(m / \mathrm{K}) z} \int_{z_{1}}^{z} e^{j \omega(m / \mathrm{K}) u} \Phi(x ; u) d u$

On peut employer d'une façon générale les variables réduites $\zeta$ et $\xi$, d'où :

$$
\mathrm{H}(j \omega)=e^{-j \xi} \int_{\zeta_{1}}^{\zeta} e^{j v} \Phi(\xi ; v) d v
$$

\section{3-7. - Surpression derrière les murs de quai due à la marée.}

Nous admettrons évidemment la marée sinusoïdale. Le mur de quai est celui schématisé sur la figure 54.

On a ici : $\mathrm{H}=\mathrm{H}_{0} \quad$ pour $x=0 ; z<-a$.

Done, $\Phi$ doil satisfaire :

à l'équation : $\quad \Delta \Phi=0$

et aux conditions : $\Phi=0$ pour $z=0 ; x>0$

$$
\begin{gathered}
\frac{\partial \Phi}{\partial n}=0 \text { pour } x=0 ; 0 \geqslant z>-a \\
\Phi=j \omega \frac{m}{\mathrm{~K}} \mathrm{H}_{0} \text { pour } x=0 ; z<-a
\end{gathered}
$$

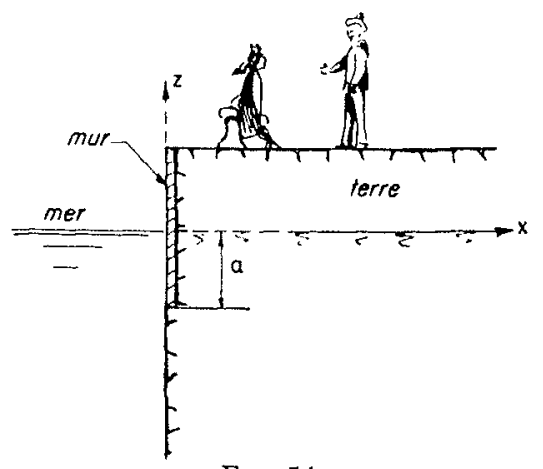

FIG. 54 
Comme dans le cas précédent, on voit directement que le champ isotherme de la fonction $Z=--\operatorname{Arg} \operatorname{sh}(x+j z) / a$ (fig. 55) donne la solution du problème.

D'où :

$$
\Phi=-j \omega \frac{m}{\mathrm{~K}} \frac{2}{\pi} \mathrm{H}_{0} \mathfrak{g}\left\{\operatorname{Argsh} \frac{x+j z}{a}\right\}
$$

$\mathscr{Y}\{\ldots\}$ signifiant : partie imaginaire de...

Et H est donnée par :

$$
\frac{\partial \mathrm{H}}{\partial z}+j \omega \frac{m}{\mathrm{~K}} \mathrm{H}=-j \omega \frac{m}{\mathrm{~K}} \frac{2}{\pi} \mathrm{H}_{0} \mathfrak{g} ; \operatorname{Arg} \operatorname{sh} \frac{x+j z}{a} ;
$$

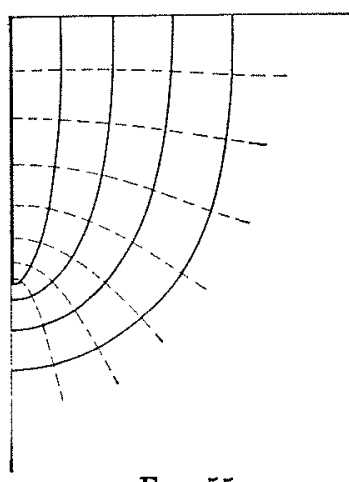

FIG. 55

D'où :

$$
\mathrm{H}=-j \omega \frac{m}{\mathrm{~K}} \frac{2}{\pi} \mathbf{H}_{0} e^{-j \omega(m / \mathrm{K}) z} \int_{0}^{z} e^{j \omega(m / \mathrm{K}) u} \mathfrak{g}\left\{\operatorname{Argsh}\left(\frac{x+j u}{a}\right)\right\} d u+e^{-j \omega(m / \mathrm{K}) \approx} f(x)
$$

On trouve $f(x)$ au paragraphe $3-6$.

Finalement, on obtient :

$$
\mathrm{H}=\mathrm{H}_{0} e^{-j(\zeta+\alpha)} e^{-\xi}-\frac{2}{\pi} j \mathrm{H}_{0} e^{-j \zeta} \int_{-\alpha}^{\zeta} e^{j v} \mathcal{F}\left\{\operatorname{Argsh} \frac{\xi+j v}{\alpha}\right\} d v
$$

en posant comme précédemment :

$$
\frac{\omega m}{\mathrm{~K}} z=\zeta ; \frac{m \omega}{\mathrm{K}} x=\xi ; \frac{m \omega}{\mathrm{K}} a=\alpha ; \frac{m \omega}{\mathrm{K}} u=v
$$

Le calcul de $\mathrm{H}(x ; z)$ est délicat dans le cas général.

Sur le mur du quai $(\xi=0 ; 0 \geqslant \zeta>-\alpha)$ on a :

$$
\mathrm{H}=\mathrm{H}_{0} e^{-j(\zeta+\alpha)}-\frac{2}{\pi} j \mathrm{H}_{0} e^{-j \zeta} \int_{-\alpha}^{\zeta} e^{j v} \operatorname{Arcsin} \frac{v}{\alpha} d \nu
$$

Compte tenu de la pression extérieure due à la mer, la force totale sur le mur est .

$$
\mathbf{F}=\varpi \int_{-a}^{0}\left(\mathrm{H}-\mathrm{H}_{0}\right) d z
$$
trouve :

F est donc donnée par une intégrale double. Si on intervertit l'ordre des intégrations, on

$$
\mathrm{F}=-\varpi \mathrm{H}_{0} a\left\lceil\frac{2}{\pi}+j \frac{1}{x}\left(1-e^{-j \alpha}\right)+\frac{2}{\pi} \int_{0}^{1} e^{-j w w} \operatorname{Arcsin} w d w\right\rceil
$$

La dernière inlégrale peut s'intégrer par parlies et on trouve :

$$
\frac{2}{\pi} \int_{0}^{1} e^{-j \alpha w} \operatorname{Arcsin} w d w=j \frac{e^{-j \alpha}}{\alpha}-\frac{\mathrm{S}_{0}(\alpha)}{\alpha}-j \frac{J_{0}(\alpha)}{\alpha}
$$

$J_{0}(\alpha)$ : fonction de Brssel d'ordre zéro.

$S_{0}(\alpha)$ : fonction de Sturve d'ordre zéro.

On en déduit :

$$
\mathrm{F}=-\varpi \mathrm{H}_{0} a\left[\frac{2}{\pi}-\frac{\mathrm{S}_{0}(\alpha)}{\alpha}+j \frac{1-\mathrm{J}_{0}(\alpha)}{\alpha}\right]
$$


On peut également calculer le moment des forces par rapport à l'origine :

$$
\mathrm{M}=\varpi \int_{-a}^{0} z\left(\mathrm{H}-\mathrm{H}_{0}\right) d z
$$

On peut calculer cette intégrale en fonction de F. Tous calculs faits, on trouve :

$$
\mathrm{M}=\varpi \mathrm{H}_{0} a^{2}\left[\frac{1}{4}-j \frac{1}{\alpha} \frac{\mathrm{F}}{\varpi \mathrm{H}_{0} a}\right]
$$

Le système des forces appliquées est équivalent à une force unique. Son point d'application peut être calculé à partir de $M$. Il varie périodiquement mais non sinusö̈dalement.

Sur la figure 29 on voit que sì $\alpha>6$ ou 8 , on peut admettre $\alpha=\infty$. D'où :

$$
\begin{aligned}
& \mathrm{F}=-\frac{2}{\pi} \varpi \mathrm{H}_{0} a \\
& \mathrm{M}=\frac{1}{4} \varpi \mathrm{H}_{0} a^{2}
\end{aligned}
$$

En pratique (marées de l'ordre de vingt-quatre heures; mur de l'ordre de $5 \mathrm{~m}$; K de l'ordre de $10^{-3}$ à $10^{-5} ; m$ de l'ordre de $5\left(^{*}\right)$ à $\left.30 \%\right) ; \alpha$ varie de quelques centièmes à quelques centaines.

Quand $\alpha$ est grand, tout revient à négliger $\partial H / \partial z$ devant $j \omega(m / K) H$ dans la condition de surface libre. Dans ce cas, on peut faire un calcul plus simple, comme on le verra plus loin.

Remarquons en passant que la fonction $\Phi$ calculée plus haut n'est pas définie d'une façon unique. On pourrait en effet admettre une singularité au point $z=-a ; x=0$; par exemple un pôle. Par une inversion et une transformation «Joukowski inverse », on obtient un champ connu (superposition d'un champ uniforme et d'un doublet (fig. 56). On pourrait donc ajouter à la solution $\Phi$ adoptée la fonction :

C : constante arbitraire.

$$
\operatorname{cg}: \frac{\sqrt{(x+j z)^{2}+a^{2}}}{x+j z} ;
$$

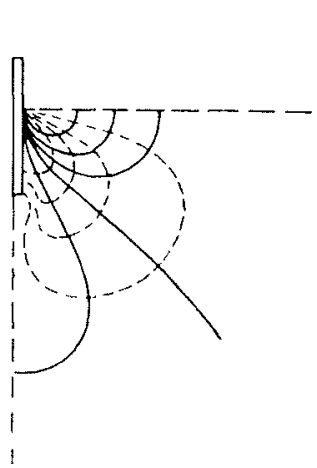

F1c. 56

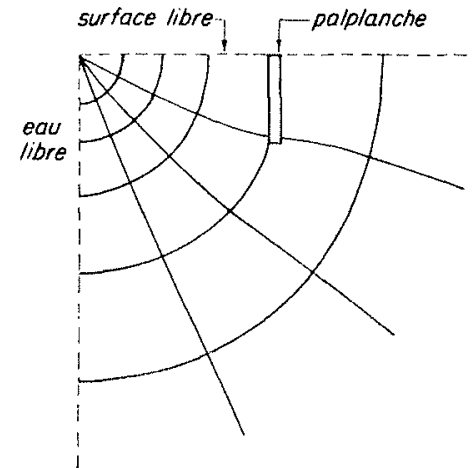

Fig $57 \mathrm{~A}$

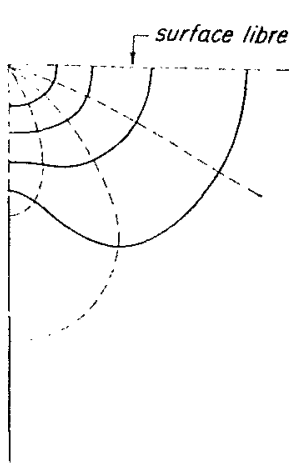

Fig. $57 \mathrm{l3}$

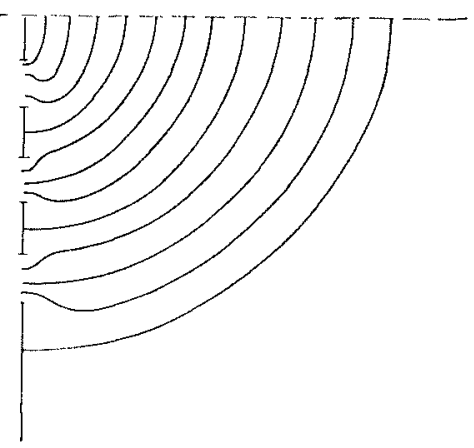

Fic. 58

Cette fonction satisfait à toutes les conditions imposées à $\mathbf{\Phi}$.

Néanmoins, si $C \neq 0$, la force sur le mur est infinie. D’une façon générale, les écoulements physiquement possibles sont ceux pour lesquels $\Phi$ n'a pas de singularité sur le contour. Alors $H$ et $\Phi$ sont déterminés d'une façon unique.

On peut également étudier par cette méthode d'autres écoulements plus compliqués, par exemple ceux schématisés sur les figures $57,57_{\mathrm{A}}$ met en jeu des fonctions ellipłiques. D'autres écoulements encore mettent en jeu des fonctions hyperelliptiques non tablées); ainsi le cas de la figure 58 (mur avec drains). Il semble impossible de prévoir exactement ce qui se passe dans ce cas; les lignes de flux partant des drains aboutissent à la surface libre, il est donc difficile de faire une approximation.

$\left.{ }^{\star}\right)$ Les sols alternativement à sec et dans l'eau ont souvent une faible porosité effective. 
Etudions maintenant rapidement quelques cas nouveaux en admettant l'approximation qui consiste à poser $\mathrm{H}=0$ à la surface libre. frontières.

Dans ce cas, le problème est classique puisqu'on connaît soit $H$, soit $\partial H / \partial n$ dans toutes les

Cette approximation «éteint » les ondes puisque $\mathrm{H}$ aura la même phase partout. Elle n'est acceptable que tout près de la frontière de la nappe et peut servir à calculer les efforts sur les ouvrages. On peut faire cette approximation quand l'ouvrage est tel que :

$a$ : dimension principale de l'ouvrage.

$$
\frac{m \omega}{\mathrm{K}} a>5 \text { ou } 10
$$

Dans cette approximation, les caractéristiques $m$ et $\mathrm{K}$ n'interviennent plus; on n'a donc pas besoin de les connaître avec précision et elles ont le droit d'être variables dans une certaine mesure. En outre, on n'a plus besoin d'admettre la profondeur infinie.

Soit d'abord la disposition schématisée sur la figure 59.

$H$ est donné par :

$$
\Delta \mathbf{H}=\mathbf{0}
$$

et :

$$
\begin{aligned}
\frac{\partial \mathrm{H}}{\partial n} & =0 \text { sur A B C } \\
\mathrm{H} & =\mathrm{H}_{0} \text { sur C D } \\
\frac{\partial \mathrm{H}}{\partial n} & =0 \text { sur D E } \\
\mathrm{H} & =0 \text { sur A F }
\end{aligned}
$$

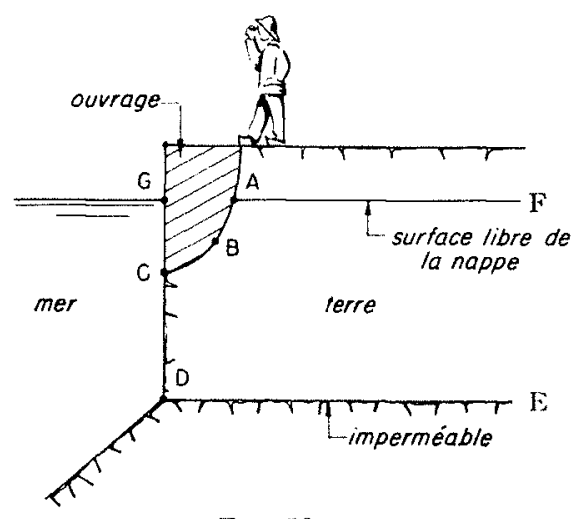

F16. 59 telle que :

Dans le cas des problèmes plans, on peut associer à la fonction harmonique $H$ la fonction $G$

$$
\frac{\partial \mathbf{G}}{\partial x}=-\frac{\partial \mathrm{H}}{\partial z} \text { et } \frac{\partial \mathbf{G}}{\partial z}=\frac{\partial \mathbf{H}}{\partial x}
$$

G est alors défini à une constante additive près. Nous ajusterons cette constante par la suite.

Alors :

$$
\mathrm{H}(x ; z)+j \mathrm{G}(x ; z)=f(x+j z)=f(\zeta)
$$

avec :

$$
x+j z=\zeta
$$

La force hydraulique exercée sur la partie A B C du mur est alors donnée par ses composantes $F_{1 x}$ et $\mathbf{F}_{1 z}$ :

$$
\begin{aligned}
& \mathrm{F}_{1 x}=\int_{\mathrm{C}}^{\mathrm{A}} p d z=\omega \int_{\mathrm{C}}^{\mathrm{A}} \mathrm{H} d z-\pi \int_{\mathrm{C}}^{\mathrm{A}} z d z \\
& \mathrm{~F}_{1 \tilde{x}}=\sigma \int_{\mathrm{O}}^{\mathrm{A}} \mathrm{H} d x-\pi \int_{\mathrm{C}}^{\mathrm{A}} z d x
\end{aligned}
$$

La force sur la partie $\mathrm{C} G$ est donnée par :

$$
\begin{aligned}
& \mathrm{F}_{2 x}=\varpi \int_{\mathrm{C}}^{\mathrm{G}} \mathrm{H}_{0} d z-\varpi \int_{\mathrm{O}}^{\mathrm{G}} z d z \\
& \mathrm{~F}_{2 z}=\varpi \int_{\mathrm{C}}^{\mathrm{G}} \mathrm{H}_{0} d x-\varpi \int_{\mathrm{C}}^{\mathrm{G}} z d x
\end{aligned}
$$


La force totale est donnée par :

$$
\begin{aligned}
& \mathrm{F}_{\infty}=\varpi \int_{\mathrm{c}}^{\mathrm{G}} \mathrm{H}_{0} d z-\varpi \int_{\mathrm{O}}^{\mathrm{A}} \mathrm{H} d z+\varpi \int_{\mathrm{G}}^{\mathrm{A}} z d z \\
& \mathrm{~F}_{z}=\varpi \int_{\mathrm{o}}^{\mathrm{Q}} \mathrm{H}_{0} d x-\omega \int_{\mathrm{a}}^{A} \mathrm{H} d x+\pi \int_{\mathrm{G}}^{A} z d z
\end{aligned}
$$

$\int_{\mathrm{G}}^{\mathrm{A}} z d z$ est visiblement nulle; $\varpi \int_{\mathrm{G}}^{\mathrm{A}} z d x$ est la poussée d'Archimède, soit $\mathrm{A}$ :

D'où :

$$
\begin{aligned}
& \mathbf{F}_{x}=\varpi \mathrm{H}_{0}\left(z_{\mathrm{G}}-z_{\mathrm{O}}\right)-\varpi \int_{\mathrm{o}}^{\mathrm{A}} \mathrm{H} d z \\
& \mathbf{F}_{z}=\varpi \mathrm{H}_{0}\left(x_{\mathrm{G}}-x_{\mathrm{C}}\right)-\varpi \int_{\mathrm{o}}^{\mathrm{A}} \mathrm{H} d x+\mathrm{A}
\end{aligned}
$$

Si on pose :

$$
\mathbf{F}=\mathrm{F}_{z}+j \mathrm{~F}_{z}-\mathbf{A}
$$

F est donné par :

$$
\mathrm{F}=\varpi \mathrm{H}_{0}\left(\zeta_{\mathrm{G}}-\zeta_{\mathrm{O}}\right)-\varpi \int_{\mathrm{c}}^{\Lambda} \mathrm{H} d \zeta
$$

Comme $(\partial H / \partial n)=0$ sur le mur la fonction $G$ y est constante. Nous choisirons la constante additive dont il était question plus haut, de telle façon que $\mathrm{G}=0$ sur le mur. Alors on $\mathbf{a}$ :

$$
\mathrm{F}=\varpi \mathrm{H}_{0}\left(\zeta_{\mathrm{G}}-\zeta_{\mathrm{O}}\right)-\varpi \int_{\mathrm{o}}^{\mathrm{A}} f(\zeta) d \zeta
$$

On peut de même calculer le moment par rapport à l'origine; il est donné par :

$$
\mathrm{M}=\mathcal{A}\left\{\frac{\varpi \mathrm{H}_{0}}{2}\left(\zeta_{\mathrm{G}}{ }^{2}-\zeta_{\mathrm{O}}{ }^{2}\right)-\varpi \int_{\mathrm{O}}^{\mathrm{A}} \zeta f(\zeta) d \zeta\right\}+\mathrm{M}_{\mathrm{A}}
$$

$\mathfrak{R}\{\ldots\}$ signifiant partie réelle de...

$M_{A}$ : moment dû à la poussée d'Archimède.

Etudions à titre d'exemple le cas schématisé sur la figure 60 .

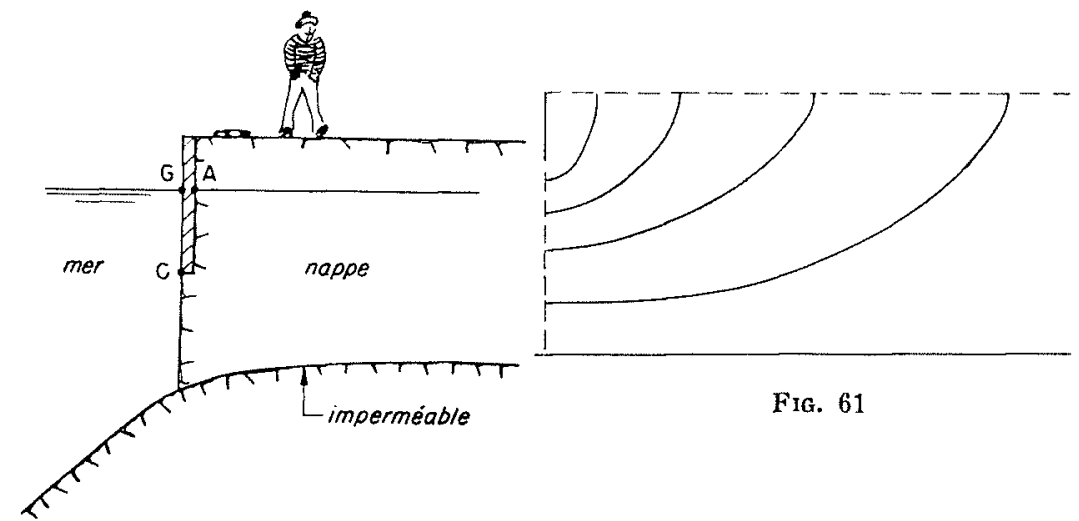

FIG. 60

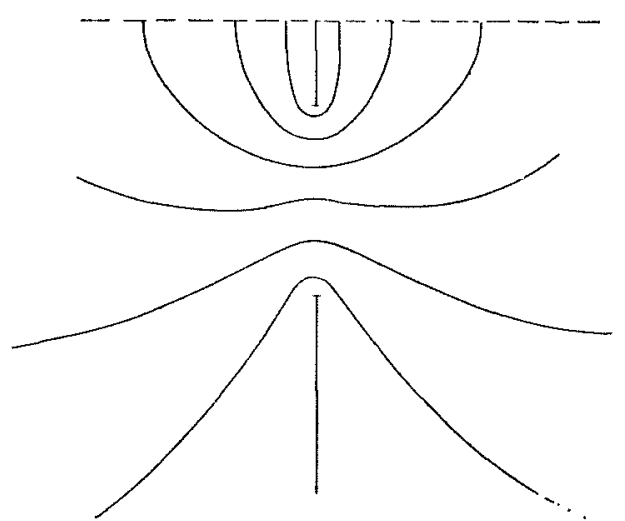

Fig. 62

Le champ isotherme de la fonction $f(\zeta)$ est donné par la figure 61.

Soit l'abscisse de $\mathrm{C}:-a$; celle de $\mathrm{D}:-\mathrm{L}$.

Par la transformation $\tau=\operatorname{sh}(\pi \zeta / 2 \mathrm{~L})$ on obtient la figure 62 qui représente le réseau isotherme de :

$$
\varphi(\tau)=\frac{\mathrm{H}_{0}}{\mathrm{~K}\left({ }^{\star}\right)} \operatorname{sn}^{-1}\left(\frac{j \tau}{k} ; k\right)
$$

( ${ }^{*} \mathrm{~K}$ est ici le quart de la période de la fonction «sn ». La perméabilité n'intervient pas dans le problème, puisqu'on a admis que $\omega \mathrm{ma} / \mathrm{K}$ était grand, donc $\mathrm{K}$ petit. 
$\mathrm{sn}^{-1}$ étant la fonction inverse de $\ll \mathrm{sn} »$.

D'où :

$$
f(\zeta)=\mathrm{H}(x ; z)+j \mathrm{G}(x ; z)=\frac{\mathrm{H}_{0}}{\mathrm{~K}} \operatorname{sn}^{-1}\left(\frac{j}{k} \operatorname{sh} \frac{\pi \zeta}{2 \mathrm{~L}} ; k\right)
$$

Pour $\zeta=0$, on a $\mathrm{H}=\mathrm{G}=0$; la constante additive est nulle.

$$
f(\zeta)=\frac{\mathrm{H}_{0}}{\mathrm{~K}} \int_{0}^{\frac{j}{k} \sin \frac{\pi \zeta}{2 \mathrm{~L}}} \frac{d u}{\sqrt{\left(1-u^{2}\right)\left(1-k^{2} u^{2}\right)}}=\frac{j \mathrm{H}_{0}}{k \mathrm{~K}} \int_{0}^{\pi \zeta / 2 \mathrm{~L}} \frac{d v}{\sqrt{1+\left(1 / k^{2}\right) \mathrm{sh}^{2} v}}
$$

en posant $u=j / k \operatorname{sh} v$.

Le point $\mathrm{C}$ est visiblement donné par $\tau=-j k$.

Done:

$$
\begin{aligned}
& \zeta_{\mathrm{C}}=-(2 / \pi) j \mathrm{~L} \text { Arc } \sin k \text { avec } k=\sin a \pi / 2 \mathrm{~L} . \\
& \zeta_{\mathrm{C}}=-j a
\end{aligned}
$$

et :

$$
\begin{aligned}
& \int_{\mathrm{a}}^{\mathrm{A}} f(\zeta) d \zeta=\frac{j \mathrm{H}_{0}}{k \mathrm{~K}} \int_{-j a}^{0} d \zeta \int_{0}^{\pi \zeta / 2 \mathrm{~L}} \frac{d v}{\sqrt{1+\left(1 / k^{2}\right) \operatorname{sh}^{2} v}} \\
& \quad=j \frac{\mathrm{H}_{0} \mathrm{~L}}{\mathrm{~K}(\pi a / 2 \mathrm{~L})} \int_{0}^{\pi a / 2 \mathrm{~L}} \frac{(2 t / \pi)-(a / \mathrm{L}) d t}{\sqrt{\sin ^{2}(a \pi / 2 \mathrm{~L})-\sin ^{2} t}} \\
& \quad \text { où }: \frac{\mathrm{F}}{j \varpi \mathrm{H}_{0} a}=1-\frac{\beta}{\mathrm{K}(\beta)} \int_{0}^{1} \frac{(1-u) d u}{\sqrt{\sin ^{2} \beta-\sin ^{2}(\beta u)}}
\end{aligned}
$$

De même :

$$
\frac{M}{a^{2} \mathrm{H}_{0} \varpi}=\frac{1}{2}-\frac{1}{2} \frac{\beta}{\mathrm{K}(\beta)} \int_{0}^{1} \frac{\left(1-u^{2}\right) d u}{\sqrt{\sin ^{2} \beta-\sin ^{2}(\beta u)}}
$$

Ces intégrales définies ne peuvent pas s'exprimer au moyen de fonctions tablées. On peut néanmoins facilement déterminer leur valeur par planimétrage. On trouve finalement $\mathrm{F} / \omega \mathrm{H}_{0} a$ et $\mathrm{M} / \omega \mathrm{H}_{0} a^{2}$ en fonction de $\beta$ (fig. 32). A partir de $(\alpha / \mathrm{L})<(2 / 3)$, (c'est-à-dire $\beta<1$ ), on peut admettre $\mathrm{L}=\infty$.

Par ce calcul et d'autres analogues, on peut facilement voir que seuls les obstacles et irrégularités situés à une distance inférieure à la taille de l'obstacle principal interviennent en ce qui concerne les forces.

Comme autre exemple, étudions le cas du mur épais (fig. 63).

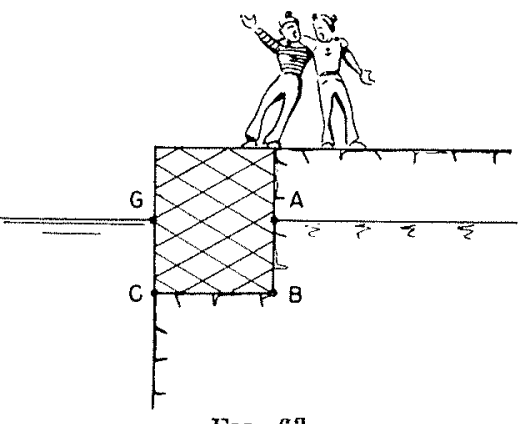

FIG. 63

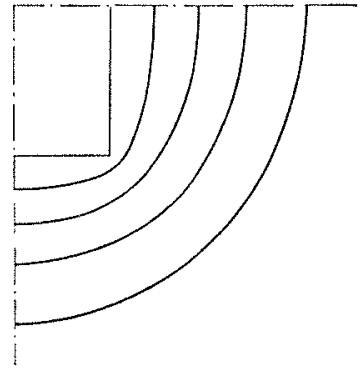

F1G. 64

Le réseau isotherme de $f(\zeta)$ est donné par la figure 64. On peut trouver son expression analytique par la méthode de l'hodographe. Celui-ci est représenté sur la figure 65. En utilisant la transformation qui fait correspondre $\log z$ à $z$, on obtient la figure 66. La fonction réciproque est représentée sur la figure 67. 
Les équations de ces réseaux sont:

Figure $67: Z=-1 / 2 \log \lambda^{\prime 2}[\sin (\tau-a) \cdot \sin (\tau+a)]$

Figure $66: \tau=\ldots-1 / 2 \log \lambda^{\prime 2}[\sin (Z-a) \cdot \sin (Z+a)]$

Figure $05: \tau=\frac{1}{\lambda^{\prime} \sqrt{\sin (Z-a) \cdot \sin (Z+a)}}$

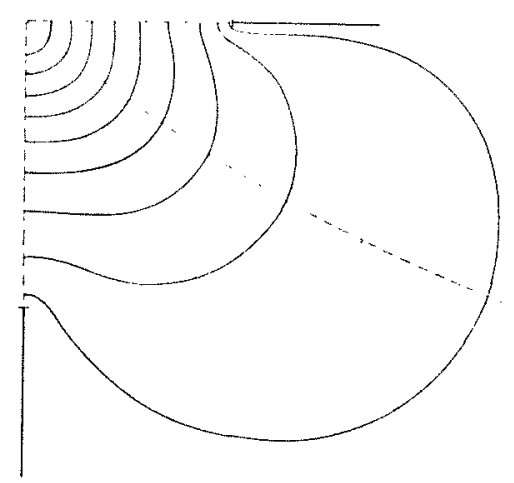

FIc. 65

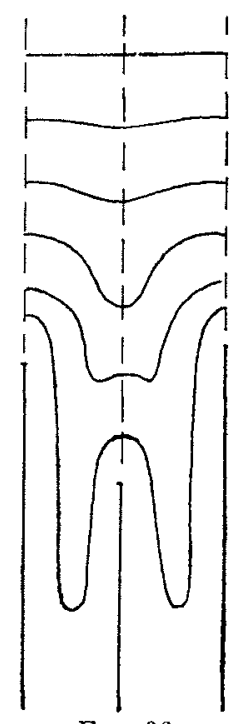

Fig. 66

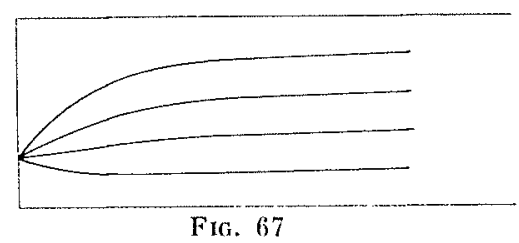

Fig. 67

L'équation de la fonction cherchée est donc:

D'où :

$$
\frac{d f}{d \zeta}=\frac{1}{\lambda^{\prime} \sqrt{\sin (u f-a)-\sin (\mu f+a)}}
$$

$$
\zeta=\lambda \int_{0}^{\mu t} d u \sqrt{\sin ^{2} u-\sin ^{2} a}+C^{\operatorname{ste}}
$$

La constante introduit une translation de $\zeta$. On peut donc la supprimer.

La correspondance des points est la suivante

$$
f=0: \zeta=0
$$

$$
\begin{aligned}
& \mu f=\varepsilon a \quad: \zeta=\varepsilon j \lambda\left[\mathrm{E}(\alpha)-\cos ^{2} a \mathrm{~K}(\alpha)\right]=\varepsilon j \mathrm{~B} \quad \text { avec } \varepsilon=-1 \\
& \mu f=\varepsilon \pi / 2: \zeta(\varepsilon \pi / 2)=\zeta(\varepsilon a)+\lambda\left[\mathrm{E}\left(\frac{\pi}{2}-a\right)-\sin ^{2} \alpha \mathrm{K}\left(\frac{\pi}{2}-a\right)\right]=\varepsilon j \mathrm{~B}+\mathrm{C}
\end{aligned}
$$

On obtient l'écoulement cherché à une symétrie près en posant :

Les longueurs sont données par :

$$
\mu=-\frac{\pi}{2 \mathrm{H}_{0}}
$$

$$
\begin{aligned}
& \mathrm{L}_{1}=\mathrm{AB}=\lambda\left[\mathrm{E}(a)-\cos ^{2} a \mathrm{~K}(a)\right] \\
& \mathrm{L}_{2}=\mathrm{B} \mathrm{C}=\lambda\left[\mathrm{E}\left(\frac{\pi}{2}-a\right)-\sin ^{2} a \mathrm{~K}\left(\frac{\pi}{2}-a\right)\right]
\end{aligned}
$$

Le rapport $\mathrm{A} B / \mathrm{BC}$ ne dépend que de la quantité « $a »$. Calculons $\mathrm{F}$ :

$$
\mathrm{F}=j \varpi \mathrm{H}_{0} \mathrm{~L}_{1}-\varpi \int_{0}^{\Delta} f(\zeta) d \zeta
$$


D'où :

$$
\begin{aligned}
& \frac{\mathrm{F}_{x}}{\omega \mathrm{L}_{1} \mathrm{H}_{0}}=1-\frac{2}{\pi\left[\mathrm{E}(a)-\cos ^{2} a \mathrm{~K}(a)\right]} \int_{a}^{\pi / 2} u \sqrt{\sin ^{2} u-\sin ^{2} a} d u \\
& \frac{\mathrm{F}_{z}}{\sigma \mathrm{L}_{2} \mathrm{H}_{0}}=\frac{2}{\pi\left[\mathrm{E}\left(\frac{2}{\pi}-a\right)-\sin ^{2} a \mathrm{~K}\left(\frac{\pi}{2}-a\right)\right]} \int_{0}^{a} u \sqrt{\sin ^{2} a-\sin ^{2} u} d u
\end{aligned}
$$

Si on pose $(A B / B C)=\rho$, on peut calculer $F_{x} /\left(\varpi L_{1} H_{0}\right)$ et $F_{z} /\left(\varpi L_{2} H_{0}\right)$ en fonction de ? seul (fig. 31 ).

\section{3-8. - Fhénomènes non sinusoïdaux dans les nappes de grande épaisseur.}

Si le niveau d'eau libre varie d'une façon non sinusoïdale dans le temps, on peut décomposer la fonction $h_{0}(t)$ donnant cette variation en spectre (ou série) de Fourier et obtenir la solution du problème par la méthode bien connue de Fourier.

Le spectre de $h_{0}(t)$ sera noté $\mathscr{H}_{0}(j \omega)$ :

$$
\mathcal{H}_{0}(j \omega)=\int_{-\infty}^{+\infty} h_{0}(t) e^{j \omega t} d t
$$

On peut résoudre les problèmes d'écoulements quelconques dans tous les cas où l'écoulement sinusoïdal est connu, c'est-à-dire en particulier dans les cas exposés au paragraphe 3-7. En reprenant les notations de ce paragraphe, on a (voir p. 26):

On en déduit :

$$
\mathrm{H}(j \omega)=j \omega \frac{m}{\mathrm{~K}} e^{-j \omega(m / \mathrm{K}) z} \int_{z_{1}}^{z} e^{j \omega(m / \mathrm{K}) u} \Phi(x ; u) d u
$$

$$
h(t)=\frac{1}{\pi} \int_{0}^{\infty} \mathcal{H}_{0}(j \omega) e^{-j \omega t} d \omega j \omega \frac{m}{\mathrm{~K}} e^{-j \omega(m / \mathrm{K}) z} \int_{\tilde{x}_{1}}^{\varepsilon} e^{j \omega(m / \mathrm{K}) u} \Phi(\boldsymbol{x} ; \boldsymbol{u}) d u
$$

On peut aussi transformer la relation (3-14) en intégrant par parties :

D’où :

$$
H(j \omega)=\Phi(x ; z)-\frac{\mathrm{K}}{m} \frac{1}{j \omega} \Phi_{z}^{\prime}(x ; z)+\left(\frac{\mathrm{K}}{m} \frac{1}{j \omega}\right)^{2} \Phi_{z^{2}}^{\prime \prime} \mp \ldots
$$

$h(t)=h_{0}(t) \Phi(x ; z)-\frac{\mathrm{K}}{m} \Phi_{z}^{\prime} \int_{0}^{t} h_{0}(t) d t+\left(\frac{\mathrm{K}}{m}\right)^{2} \Phi_{z^{\prime \prime}} \int_{0}^{t} d t \int_{0}^{t} h_{0}(t) d t \pm \ldots$

Ainsi, si : $h_{0}(t)=\mathrm{H}_{1} \Upsilon(t)$, on obtient :

$$
h(t)=\mathrm{H}_{1} \Phi\left(x ; z-\frac{m}{\mathrm{~K}} t\right)
$$

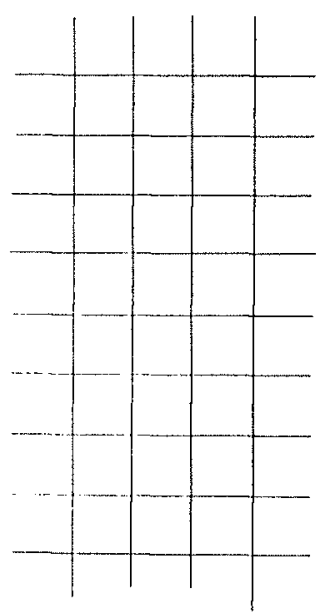

FIG. $68 a$

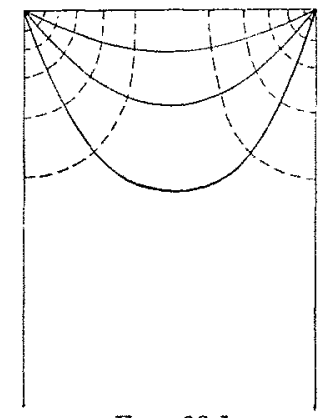

FIG. $68 b$

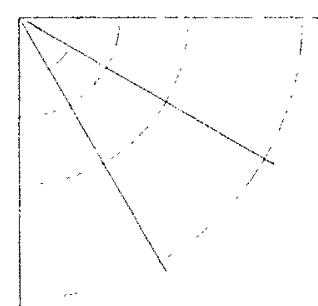

Fic. $68 c$

nombre très limité de cas. On voit de suite que cela ne peut se produire que si $H$ ou $\partial H / \partial n$ a une valeur constante sur chaque limite verticale. Il n'existe que trois cas où cela est vérifié. Ils sont schématisés sur la figure 68. Le troisième n'a aucune valeur pratique. Les deux autres donnent: 
$h=\mathrm{H}_{1} \frac{2}{\pi} \operatorname{Arctg} \frac{z-(\mathrm{K} / m) t}{x}$

et :

$h=\mathrm{H}_{1} \frac{2}{\pi}\left\{\operatorname{Arctg}\left[\operatorname{th}\left(z-\frac{\mathrm{K}}{m} t\right) \operatorname{ctg}(x-a)\right]-\operatorname{Arctg}\left[\operatorname{th}\left(z-\frac{\mathrm{K}}{m} t\right) \operatorname{ctg}(x+a)\right]\right\}$

Dans tous les autres cas, il convient d'employer la formule 3-14. Pratiquement, il n'existe pas de cas simples où $h(t)$ puisse être exprimé au moyen de fonctions tablées. Par contre, on peut chercher les forces et moments sur certains ouvrages. Ainsi pour le mur de la figure 54, on obtient à partir de :

$$
\begin{gathered}
\mathrm{F}(j \omega)=\mathcal{H}_{0}(j \omega) \varpi a\left[\frac{2}{\pi}+\frac{j}{\alpha}\left(1-e^{-j a}\right)+\frac{2}{\pi} \int_{0}^{1} e^{-j a v} \operatorname{Arcsin} v d v\right] \\
\text { avec }: \alpha=\frac{\omega m a}{\mathrm{~K}} \\
\mathrm{~F}(t)=\omega a\left[\frac{2}{\pi} h(t)-\frac{\mathrm{K}}{a m} \int_{t-(a m / \mathrm{K})}^{t} h_{0}(u) d u+\frac{2}{\pi} \int_{0}^{1} h_{0}\left(t-\frac{a m}{\mathrm{~K}}\right) \operatorname{Arcsin} v d v\right] \\
\text { Si } h_{0}(t)=\mathrm{H}_{1} \mathrm{Y}(t) \text {, c'est-à-dire pour un abaissement brusque du plan d'eau libre, on a (fig. 33) : } \\
\mathrm{F}(t)=a \mathrm{H}_{1}\left[\frac{2}{\pi} \sqrt{1-\tau^{2}}-\tau+\frac{2}{\pi} \tau \operatorname{Arcsin} \tau\right] \text { pour } t \leqslant \frac{a m}{\mathrm{~K}} \\
\mathrm{~F}(t) \equiv 0 \quad \text { pour } t>\frac{a m}{\mathrm{~K}} \\
\text { avec }: \tau=\frac{t \mathrm{~K}}{a m}
\end{gathered}
$$

On voit que la force tombe rigoureusement à zéro au bout d'un temps fini, alors que l'écoulement persiste jusqu'à $t=\infty$.

\section{3-9. - Conclusions de la III $^{\mathrm{e}}$ partie.}

Nous avons essayé d'appliquer à la théorie des nappes souterraines un certain nombre de connaissances de la physique mathématique, et plus spécialement de « l'hydraulique mathématique ». Les bases de nos études dont les équations linéarisées du paragraphe 3-1. Ces iquations sont assez proches des équations régissant les éconlements des fluides parfaits dans l'approximation de la «houle de Stokes». Seule la condition à la surface libre est différente : $\partial^{2} \Phi / \partial t^{2}$ est remplacée par $\partial \Phi / \partial t$. Cette ressemblance permet l'application d'un certain nombre de procédés mathématiques qui ont été spécialement concus pour l'hydraulique, ainsi les méthodes employées aux paragraphes 3-2, 3-6 et 3-7. Du point de vue physique, par contre, il n'y a aucune analogie profonde : le terme $\partial^{2} \Phi / \partial t^{2}$ représente une inertie, donc une énergie cinétique qui peut à tout moment se retransformer en énergie potentielle; le terme $\partial \Phi / \partial t$ représente au contraire une résistance dissipant de l'énergie. La houle est un écoulement sans perte d'énergie, tandis que les écoulements en milieu poreux sont des écoule- ments avec de très grosses pertes d'énergie et sans inertie : aussi beaucoup de phénomènes physiques de la houle n'ont pas de pendant en ce qui concerne les écoulements en milieu poreux. Ainsi, par excmple, les ondes de Mach, les phénomènes de résonance, les vagues de pente, etc...

Par ailleurs, il a été reconnu une fois de plus qu'une approximation du genre «Dupur's » est assez bonne dans le cas des nappes, seules les régions très voisines des limites ou des grosses irrégularités sont mal représentées. Il suffit le plus souvent de changer les conditions aux limites pour obtenir de bons résultats. Il est évident que les nouvelles conditions à adopter ne peuvent pas être déterminées par un calcul basé sur l'approximation de DupurT; par contre, d'autres approximations peuvent être faites, comme le montre le cas des murs de quai. Rappelons également que les approximations du genre Dupvit n'ont aucun sens dans le cas des écoulements à travers les digues ou d'autres écoulements pour lesquels les rapports

$$
\frac{\text { Distances horizontales }}{\text { Distances verticales }}
$$


ne sont pas au moins de l'ordre de 5 ou de 10 . (Dans le cas des nappes, ces rapports sont en général au moins de l'ordre de 30 , souvent de 100 et plus.)

En ce qui concerne les questions encore en suspens, il semble que les trois suivantes soient du plus grand intérêt :

a) En se basant sur la ressemblance mathématique avec la houle, il serait utile d'étudier une approximation meilleure que la linéarisation. Pour la houle, cette approximation est celle qui conduit aux « ondes cnoïdales ». Une telle étude a déjà été fortement développée par M. JAEGER [4]. mais se heurte dans la plupart des cas à de grosses difficultés mathématiques;

b) II conviendrait d'étudier l'équation $(3 ; 11)$ d'une facon plus approfondie que nous l'avons fait ici;

e) II conviendrait d'étudier plus exactement les conditions aux limites à imposer à l'équation $(3 ; 11)$.

Néanmoins, on voit que les mathématiques classiques sont rapidement insuffisantes. Il conviendrait probablement d'étudier numériquement un certain nombre de problèmes en faisant varier les paramètres. Ces études ne peuvent malheureusement être effectuées qu'avec de puissantes machines à calculer arithmétiques.

\section{BIBLIOGRAPHIE}

[1] Polubarinova Котchina. - Полубаринова - Кочина.Теория Движения Грунтових Вод" Gostelhizdat, Moscou (1952).

[2] Musirat. - « The flow of homogeneous Fluids through porous media 》. J. W. EDwardos Inc. Ann Arbor Michigan (1946).

[3] Tison. - « Cours d'hydraulique », $2^{\circ}$ partic, Gand, 1950.

[4] JaEgen. - « Hydraulique technique » (traduction de Mme M. Laronde). Dunod, 1954, Paris.

[5] Jahnke-Emde. - \& Tables of Functions ». Dover Publications (1945), New-York.
[6] Friedrichs. - Appendice intitule « On the Derivation of the Shallow Water Theory » a l'article : «The formation of Breakers and Bores $\gg$ de Mr. J. SToker dans Communications on Applied Mathematics, vol. $1, \mathrm{n}^{\circ} 1, \mathrm{pp} .81$ et suivantes.

[7] Boussineso. - «Esai sur la théorie des eaux courantes 》 dans les «Mémoires présentés à l'Aeadémie des Sciences ». Sciences mathématiques et physiques. Tome $23^{\circ}$, pp. 252 et suivantes. Imprimerie Nationale, Paris (1877).

[8] Fritz John. - " Waves in the presence of an inclined barrier 》 publie dans Communications on Applied Mathematics, vol. $1, \mathrm{n}^{\circ} 2$, pp. 149 et suivantes.

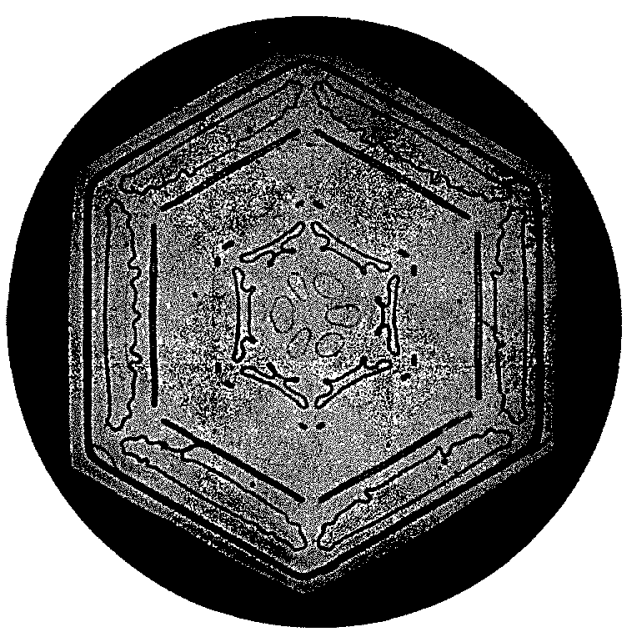

